\title{
Can the polarization tagging of the ionogram trace deceive autoscaling methods? The Learmonth case
}

\author{
Michael Pezzopane and Carlo Scotto \\ Istituto Nazionale di Geofisica e Vulcanologia, Roma, Italy
}

\begin{abstract}
This paper focuses on the problem of invalid $\mathrm{O} / \mathrm{X}$ polarization tagging of an ionogram and how this can affect ionogram autoscaling methods. To illustrate this problem, 623 ionograms recorded in March and April 2004 (days $80-105)$ by the digisonde 256 installed at Learmonth $\left(22.3^{\circ} \mathrm{S}, 114.1^{\circ} \mathrm{E}\right)$ were considered. These ionograms, often characterized by very unreliable $\mathrm{O} / \mathrm{X}$ polarization tagging of the echoes because of unresolved antenna issues, have been autoscaled by both ARTIST 4.2 and Autoscala. Results of comparisons between automatically and manually scaled foF 2 data are shown for both programs, considering as acceptable an autoscaled value that lies within $0.5 \mathrm{MHz}$ of the manual value. Autoscala values of $f o F 2$ agree with the manually-scaled values for $\sim 99 \%$ of ionograms, while ARTIST values of foF 2 agree with the manually-scaled values for $~ 75 \%$ of ionograms. While ARTIST was coded on the assumption of valid polarization tagging, the fact remains that it produces invalid results when equipment issues cause invalid tagging. Autoscaling procedures that do not use the polarization tagging will generally work better than ARTIST in such cases. However, these other procedures are susceptible to failure in other situations.
\end{abstract}

Key words Ionospheric Monitoring - Ionograms ARTIST - Autoscala

\section{Introduction}

With the advent of new technologies a digital ionosonde is able to separate the recorded information of the ordinary and extraordinary waves in terms of polarization. For instance the digisonde 256 produced by the University of Massachusetts, Lowell, using a cross antenna system, is able to code the echo amplitude recorded by the receiver and consequently rec-

Mailing address: Dr. Michael Pezzopane, Istituto Nazionale di Geofisica e Vulcanologia, Via di Vigna Murata 605, 00153 Roma, Italy; e-mail pezzopane@ingv.it ognize the polarization of the ordinary and extraordinary wave. This is evident in the digisonde ionogram pictures where the ordinary ray is depicted in red while the extraordinary ray is depicted in green. This polarization tagging of the ionogram trace represents an important aid for autoscaling methods, like ARTIST (Reinisch and Huang, 1983; Reinisch et al., 2005; McNamara, 2006), that rely on this information. Nevertheless, in certain cases errors in the polarization tagging can occur and these autoscaling systems may be deceived, with a consequent incorrect identification of the ordinary trace. Other autoscaling methods, like Autoscala (Scotto and Pezzopane, 2002, 2007; Pezzopane and Scotto, 2004, 2005) or the empirical orthogonal function method developed by Ding et al. (2007), do not rely on this tagging and provided both the ordinary and the extraordinary traces are visible give a correct elaboration of the ionogram trace. In this work we investigate this is- 
sue, focusing our attention on a dataset of ionograms recorded at Learmonth $\left(22.3^{\circ} \mathrm{S}, 114.1^{\circ}\right.$ $\mathrm{E})$, where many cases of unreliable $\mathrm{O} / \mathrm{X}$ polarization tagging are observed.

\section{The Data Analysis}

To illustrate the problems of $\mathrm{O} / \mathrm{X}$ polarization tagging, a dataset of 623 hourly ionograms recorded in March and April 2004 (days 80$105)$ by the digisonde 256 installed at Learmonth, often characterized by very unreliable $\mathrm{O} / \mathrm{X}$ polarization tagging of the echoes because of unresolved antenna issues, were considered. These ionograms, autoscaled by ARTIST 4.2 (here abbreviated to ARTIST), were also autoscaled by Autoscala. Autoscala uses ionograms in RDF file format as input (Pezzopane, 2004) and so, before applying Autoscala to ionograms recorded by the digisonde, a format change is necessary. In this change of format, in which the echo amplitude received by the digisonde is retrieved indirectly from the colour pixel depth of the ionogram picture produced by ARTIST, the information on polarization is removed, as shown in fig. 1.

With reference to the processing dataset of 623 ionograms, the following four subsets were considered:

1. subset $\mathrm{C}$ (definite values). Ionograms for which the operator was able to scale $f o F 2$ as a definite value, using neither descriptive nor qualificative letters.

2. subset D (doubtful traces). Ionograms for which the operator scaled $f o F 2$ as a doubt- ful value. For these ionograms the URSI standard suggests reporting the numerical value followed by the qualifying letter $U$ and the appropriate descriptive letter ( $\mathrm{S}$ for interference or $\mathrm{R}$ for absorption).

3. subset F (spread F). Ionograms for which a spread F condition was observed. For these ionograms the URSI standard suggests reporting the numerical value followed either by the qualifying letter $U$ and the appropriate descriptive letter $\mathrm{F}$ or by the descriptive letter F only.

4. subset I (impossible). Ionograms for which the operator was not able to observe the F2 trace for different reasons.

For subsets C, D, and F correct behaviour of the programs is assumed for ionograms scaled with acceptable values (an acceptable value is considered to lie within $\pm 0.5 \mathrm{MHz}$ of the manual value). For subset I the correct behaviour of the programs is to discard the ionograms, giving no data as output.

The automatically scaled foF 2 values produced by ARTIST and Autoscala were compared with those hand-scaled by the IPS Radio and Space Services, Australia. The results of this data analysis are reported in table I, where it appears that Autoscala performs better than ARTIST 4.5 for subsets C, and F. This is because many ionograms of these subsets are affected by an unreliable $\mathrm{O} / \mathrm{X}$ polarization tagging of the ionogram trace and this may deceive the ARTIST algorithm, as shown in figs. 2a, and $3 \mathrm{a}$, where the extraordinary ray is identified as the ordinary with a consequent overestimation of the real value of $f o F 2$; in table I the 143 cases of subset $\mathrm{C}$ resulting not acceptable for

Table I. The manually scaled foF 2 values are compared to the automatically scaled values obtained by $\mathrm{Au}$ toscala and ARTIST. The comparison is performed separately for subsets C, D, F, and I. The numbers of Acceptable values (Acc), Not Acceptable values (Not Acc), Not Scaled ionograms (NS), and Scaled ionograms (S), are shown. An acceptable value is considered to lie within $\pm 0.5 \mathrm{MHz}$ of the manual value.

\begin{tabular}{lccccccccccc}
\hline \hline & \multicolumn{3}{c}{ Subset C } & \multicolumn{3}{c}{ Subset D } & \multicolumn{3}{c}{ Subset F } & \multicolumn{3}{c}{ Subset I } \\
& Acc & Not Acc & NS & Acc & Not Acc & NS & Acc & Not Acc & NS & NS & S \\
\hline Autoscala & 598 & 0 & 0 & 5 & 0 & 0 & 16 & 2 & 0 & 2 & 0 \\
ARTIST & 454 & 143 & 1 & 2 & 3 & 0 & 5 & 13 & 0 & 2 & 0 \\
\hline
\end{tabular}


STATION YYYY DAY DDD HHHY P1 FFS S AXN PPS IGA PS Learnonth 2004 Mar21 0810000 HHI 400-1 8ic5 100 +2+ H1
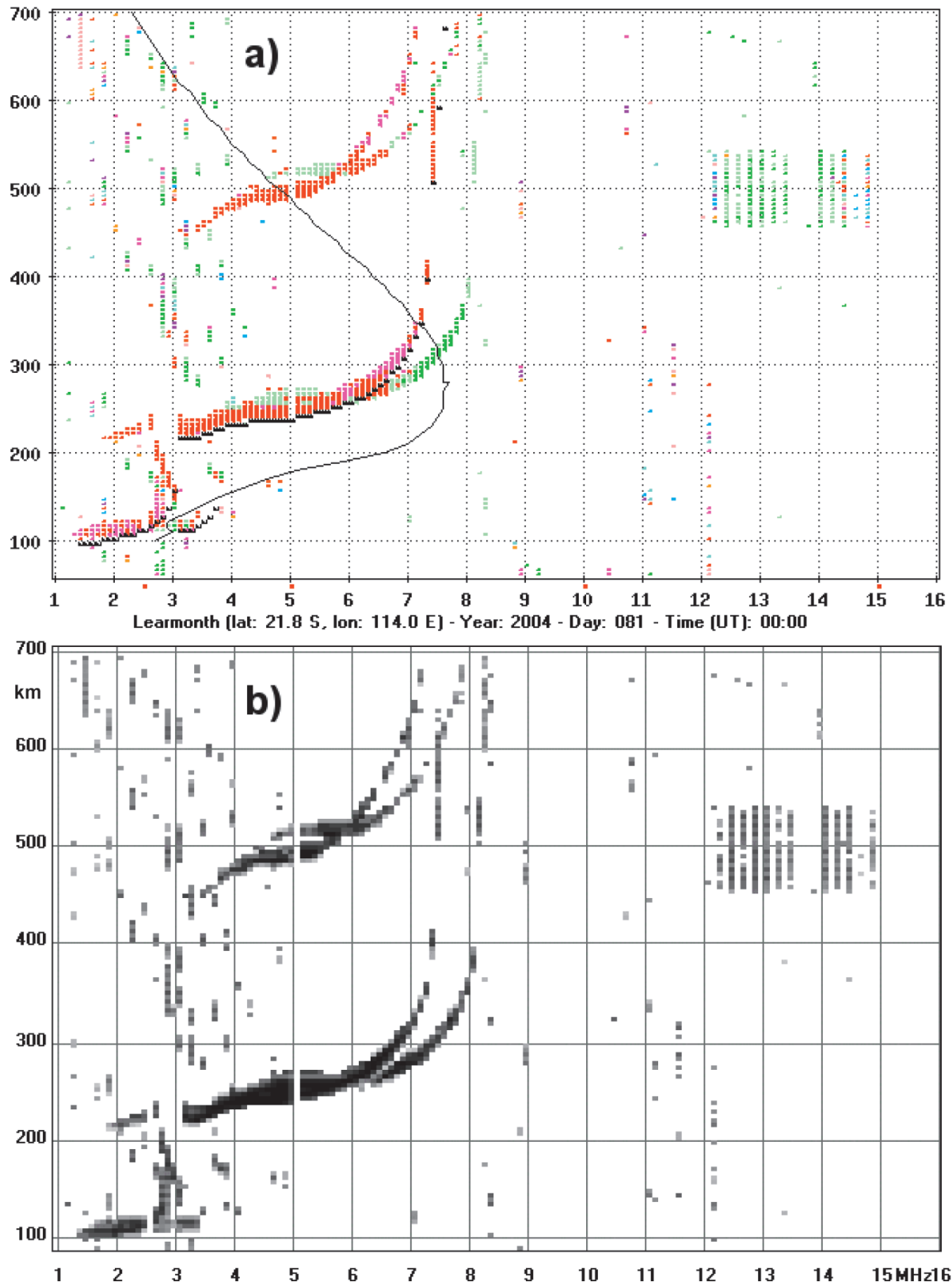

Fig. 1a,b. a) The ionogram recorded on 21 March 2004 at 00:00 UT by the digisonde 256 installed at Learmonth, and b) as it appears after the format change in which the information on polarization was removed. 
STATION YYYY DAY DDD HHMI P1 FFS S AXN PPS IGA PS Learnonth 2004 Mar22 0820300 Mill 400-1 8c5 100 +2+ A1

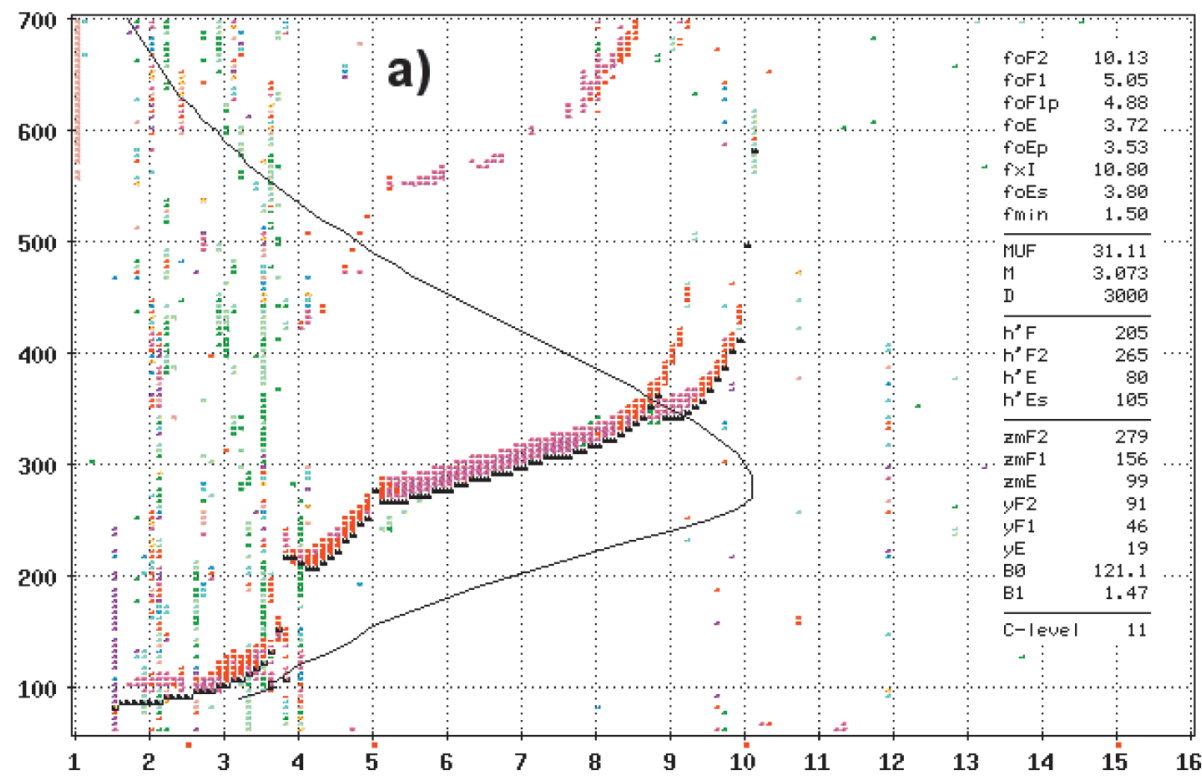

Learmonth [lat: 21.8 S, lon: 114.0 E] - Year: 2004 - Day: 082 - Time [UT]: 03:00

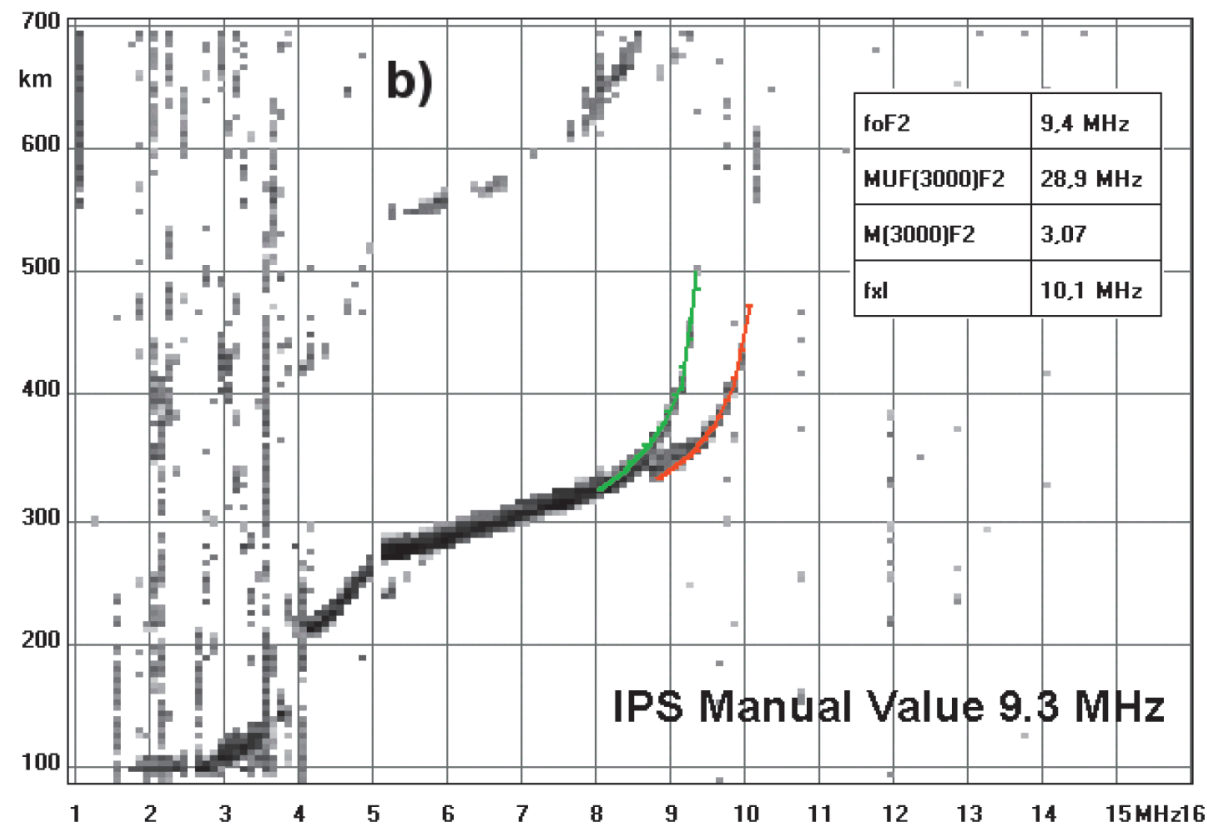

Fig. 2a,b. Ionogram recorded on 22 March 2004 at 3:00 UT by the digisonde 256 installed at Learmonth, autoscaled by a) ARTIST and b) Autoscala (in green and in red the ordinary and the extraordinary traces respectively identified by the software). In b) we also give the corresponding IPS manual value as a simple indicator of the autoscaling quality. 
STATION YYY DAY DOD HHHH P1 FFS 5 AXM PPS IFA PS Learnonth 2004 Apr02 0931900 Hill 400-1 8c5 100 +2+ H1

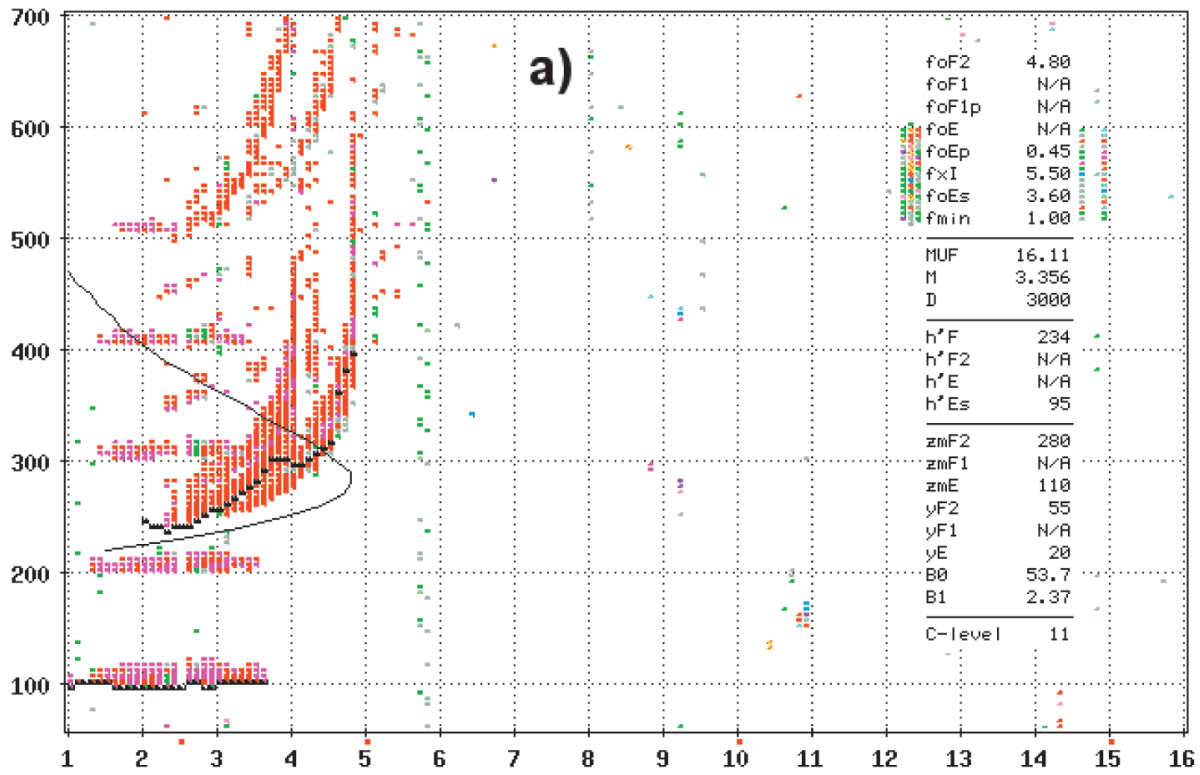

Learmonth (lat: 21.8 5, lon: 114.0 E] - Year: 2004 - Day: 093 - Time [UT]: 19:00

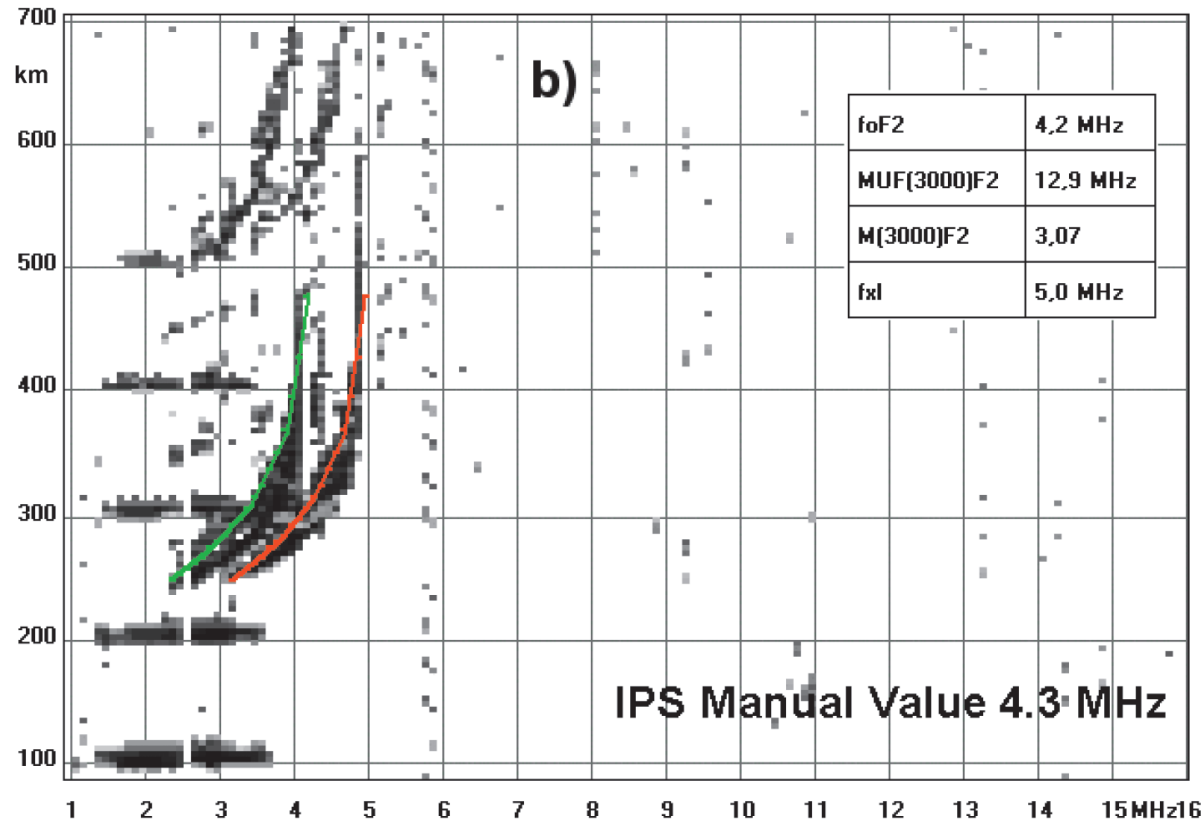

Fig. 3a,b. Ionogram recorded on 02 April 2004 at 19:00 UT by the digisonde 256 installed at Learmonth, autoscaled by a) ARTIST and b) Autoscala (in green and in red the ordinary and the extraordinary traces respectively identified by the software). In b) we also give the corresponding IPS manual value as a simple indicator of the autoscaling quality. 
ARTIST are just cases of ionograms for which this overestimation exceeds the manual value of $0.5 \mathrm{MHz}$. Nevertheless there are also cases of ionograms characterized by an incorrect polarization tagging for which ARTIST scaled the ionogram properly, as shown in fig. 4. Autoscala, not relying on this tagging, when the F2 ionogram trace is clearly visible (subset $\mathrm{C}$ ) or affected by a spread $\mathrm{F}$ condition (subset F), gave as output reliable values of $f o F 2$, as illustrated in figs. $2 b$, and $3 b$.

\section{Discussion}

Figures $2 \mathrm{a}$ and $3 \mathrm{a}$ illustrate ionograms characterized by misleading polarization tagging, with the ordinary and the extraordinary rays being depicted by the same red colour. ARTIST in these cases tends to identify the extraordinary ray as the ordinary one. This is because the ARTIST system identifies all the red points as belonging to the ordinary ray. The F region ordinary baseline defined by Reinisch and Huang (1983) is constructed by sliding a search window from the centre of the $\mathrm{F}$ trace towards higher frequencies until it finds all red points. On the other hand, the only criterion by which Autoscala differentiates the ordinary from the extraordinary ray is that the asymptote of the empirical curve used to identify the ordinary trace has to correspond to a smaller frequency than the asymptote of the empirical curve used to identify the extraordinary trace (Pezzopane and Scotto, 2007). Thus it is not possible for Autoscala to do an identification of the trace like those performed by ARTIST in fig. 2a, and 3a. Referring to the ionogram of fig. $2 \mathrm{a}$, for example, if Autoscala had carried out the same autoscaling performed by ARTIST, the two curves used by Autoscala to identify the two rays of the F2 trace, the ordinary and the extraordinary, would have been positioned as in fig. 5; but in this curves positioning Autoscala

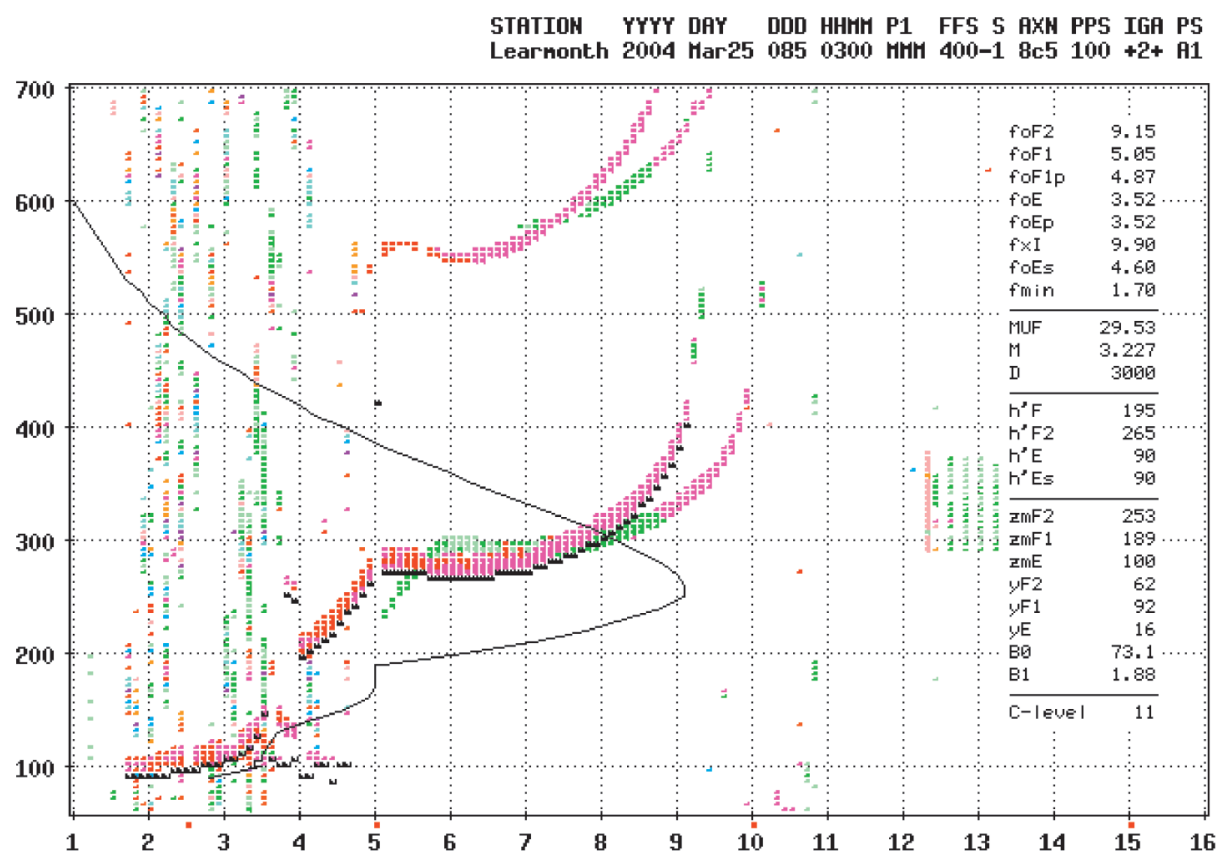

Fig. 4. Ionogram recorded on 25 March 2004 at 03:00 UT by the digisonde 256 installed at Learmonth. Although the O/X polarization tagging of the echoes is unreliable ARTIST correctly scales this ionogram. 


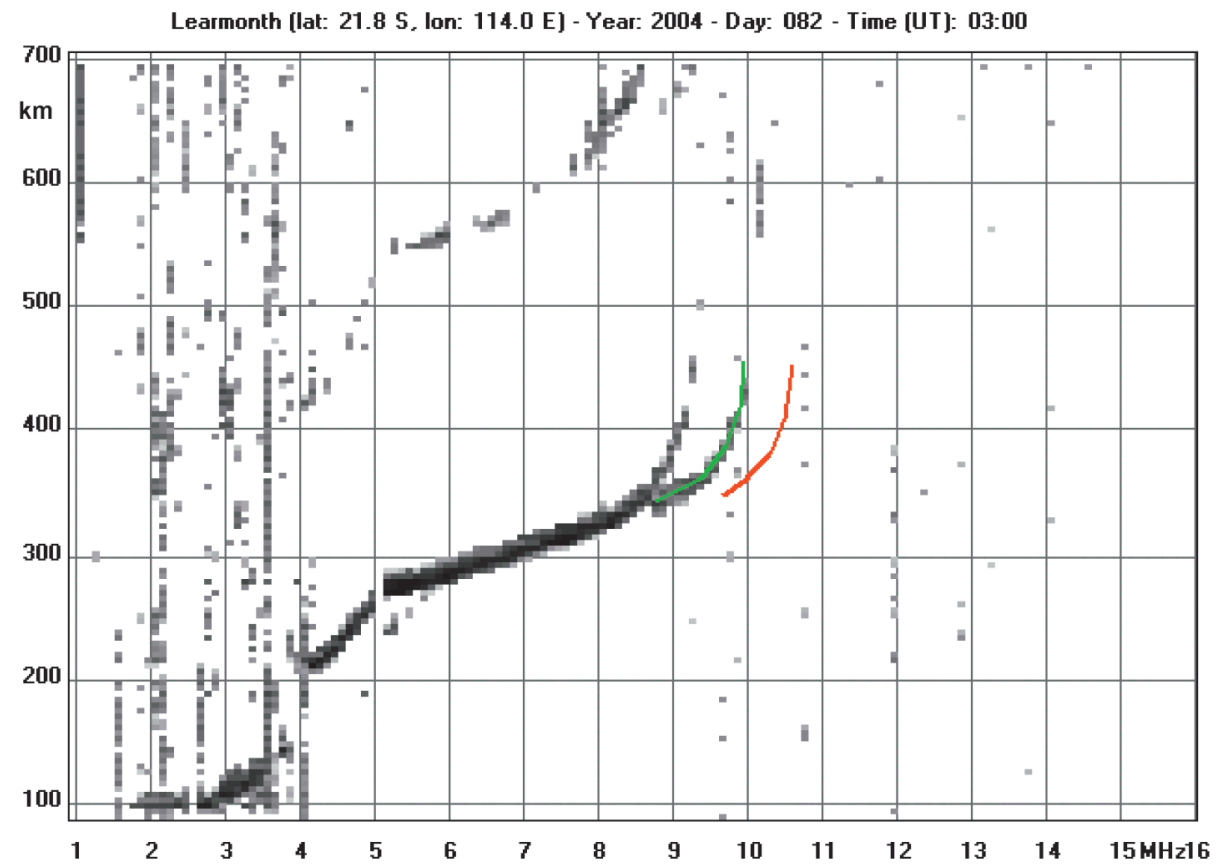

Fig. 5. The same ionogram as in fig. 2 for which Autoscala (in green and in red the ordinary and the extraordinary traces respectively identified by the software) performed the same autoscaling of ARTIST identifying the extraordinary ray of the ionogram as the ordinary one. In this case Autoscala considers the ionogram information insufficient to provide a numerical value for $f o F 2$.

would consider the ionogram information not sufficient to output a numerical value for $f o F 2$ because only one empirical curve has a significant correlation with the ionogram echo.

Figure 6 shows hourly foF 2 plots of 01 and 14 April 2004 as obtained by the ionograms autoscaled by ARTIST and Autoscala. All the ionograms of both days belong to subset $\mathrm{C}$ and the $\mathrm{O} / \mathrm{X}$ polarization tagging of the $\mathrm{F} 2$ trace was unreliable from 15 to 22 UT. Comparing manually and autoscaled values it appears that during this time ARTIST overestimated the foF 2 value by about half the gyro-frequency.

While Autoscala can work properly in the absence of polarization tagging if the ordinary and extraordinary traces are separate and pretty well defined, it is less successful when a spread F condition characterizes the ionogram. Wakai et al. (1987) stated that if echo spreading is seen for both components of the $\mathrm{F}$ trace and if the outer edge (the higher side of frequency) of the ordinary component is observed clearly, this should be regarded as the main trace from which foF $_{2}$ must be scaled. Figure 7 illustrates a case of an ionogram of this type, for which both ordinary and extraordinary components of the $\mathrm{F}$ trace are in a spread condition. In this case for ARTIST the outer edge of the ordinary trace is well marked and hence easily traceable. On the contrary, as shown in fig. $7 \mathrm{~b}$, for Autoscala it is impossible to establish whether the outer edge of the ordinary trace is clearly defined or not and as recommended by Wakai et al. (1987) the foF 2 value is scaled considering the inner edge of the trace. However, table I shows that the behaviour of Autoscala for spread F conditions is still acceptable.

It is worth noting that autoscaling methods that do not rely on the polarization information, such as Autoscala, may find problems in scaling 

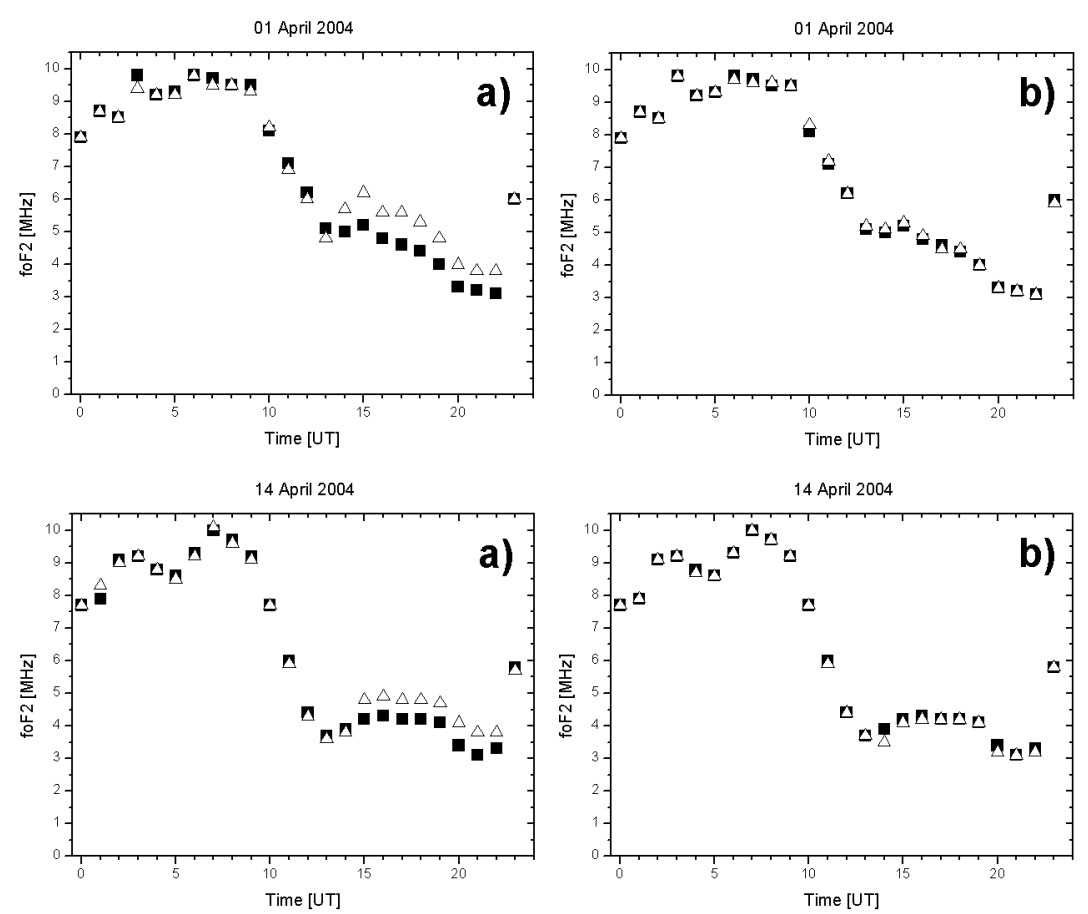

Fig. 6. Hourly foF 2 plots of 01 and 14 April 2004 as obtained by the ionograms recorded by the digisonde 256 installed at Learmonth, autoscaled by a) ARTIST and b) Autoscala. Manually and autoscaled value are indicated by solid squares and open triangles, respectively. The O/X polarization tagging of the $\mathrm{F} 2$ trace was unreliable from 15 to 22 UT. During this time ARTIST overestimated the foF 2 value by about half the gyro-frequency.

ionograms for which only the ordinary ray is clearly defined while the extraordinary one is practically absent or vice versa. To illustrate this case, fig. 8 shows an ionogram for which almost all of the extraordinary ray was artificially truncated and then processed by Autoscala. In this case, even if the ordinary ray is well defined Autoscala gives as output N/A for foF 2 because only one empirical curve (and not both) has a significant correlation with the ionogram.

\section{Conclusions}

The information on the ordinary and extraordinary wave polarization can certainly provide significant help for autoscaling methods. Nevertheless this work has pointed out that, for ionogram traces characterized by unreliable
$\mathrm{O} / \mathrm{X}$ polarization tagging, this information may be misleading. In fact when scaling ionograms characterized by unreliable polarization tagging, methods relying mainly on the $\mathrm{O} / \mathrm{X}$ polarization tagging may overestimate the real foF 2 critical frequency. In order to perform a reliable autoscaling of the ionogram trace, the polarization information of received echoes should be exploited in conjunction with other constraints. For instance one of these constraints could be that a part, even if hardly visible, of both rays has to be present on the ionogram; otherwise an autoscaling method can be easily deceived with a consequent wrong identification of the extraordinary ray as the ordinary, causing an overestimation of $f_{o} F 2$. On the other hand, autoscaling programs such as Autoscala that do not take advantage of polarization tagging are susceptible to incomplete or missing $\mathrm{O} / \mathrm{X}$ traces. 
STATION YYYY DAY DDD HHHY P1 FFS 5 AXN PPS IGA PS Learnonth 2004 Apro2 0931800 HIM 400-1 8c5 100 +2+ A1
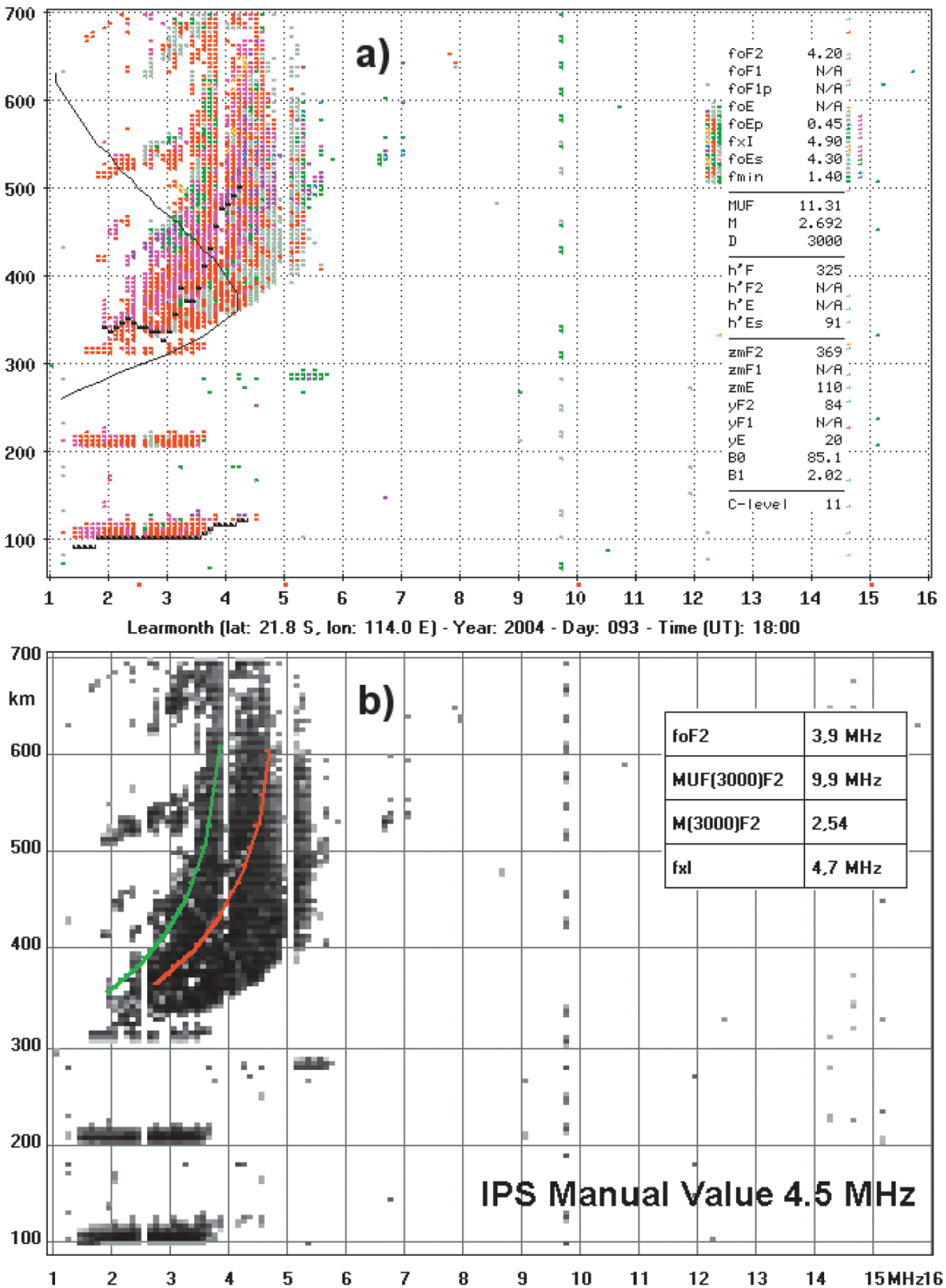

Fig. 7a,b. Ionogram recorded on 02 April 2004 at 18:00 UT by the digisonde 256 installed at Learmonth, autoscaled by a) ARTIST and b) Autoscala (in green and in red the ordinary and the extraordinary traces respectively identified by the software). In b) we also give the corresponding IPS manual value as a simple indicator of the autoscaling quality. 
STATION YYYY DAY DDD HHH P1 FFS S AXN PPS IGA PS Learnonth 2004 Har24 0840600 HHI 400-1 8c5 100 +2+ H1
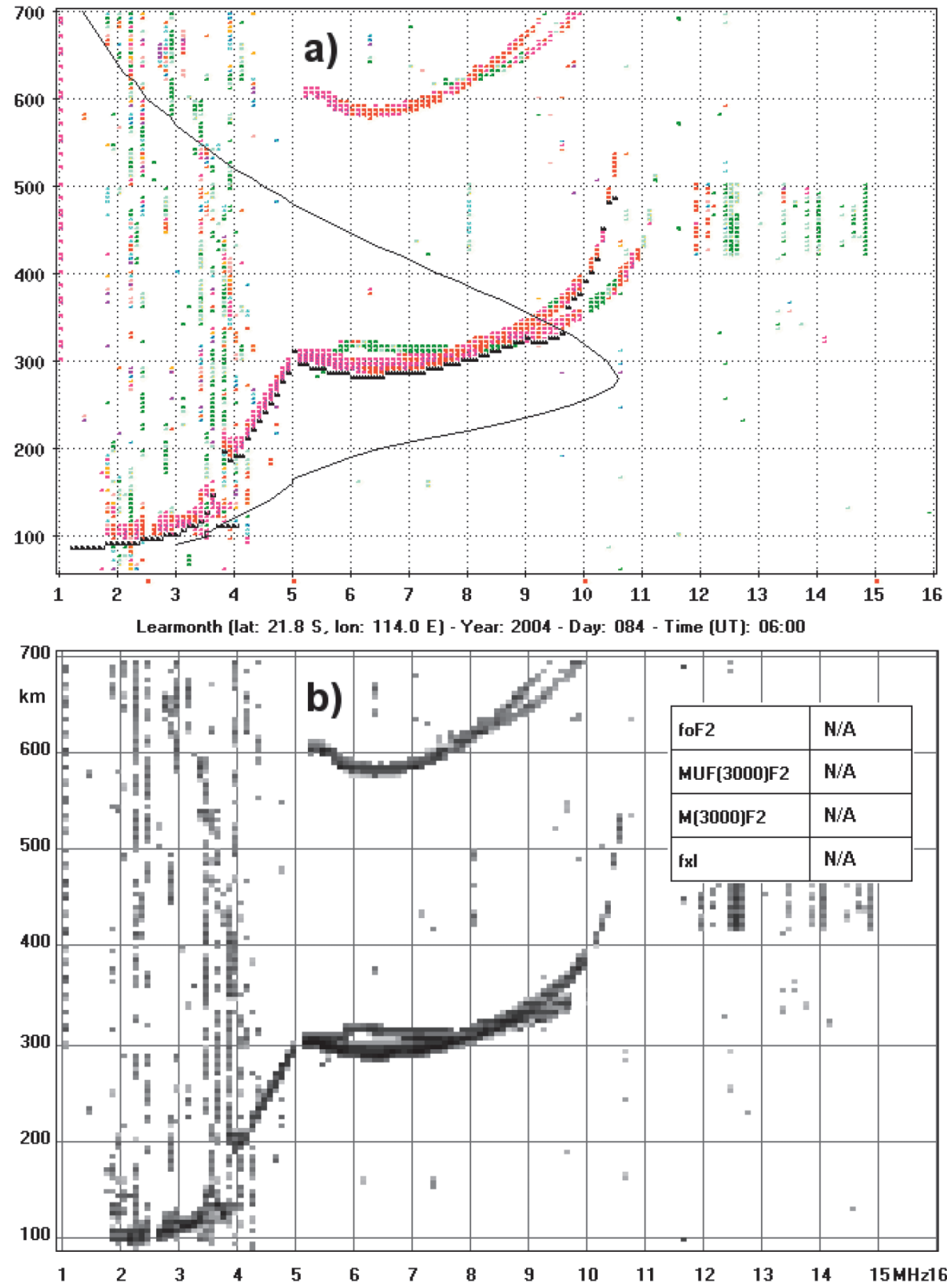

Fig. 8a,b. a) The ionogram recorded on 24 March 2004 at 06:00 UT by the digisonde 256 installed at Learmonth. b) The same ionogram in which an artificial truncation of the extraordinary ray was performed and then autoscaled by Autoscala. In this case Autoscala considered the digital information in the ionogram to be insufficient to provide a reliable value of $f o F 2$. 


\section{REFERENCES}

Ding, Z., B. Ning, W. WAN and L. LiU (2007): Automatic scaling of F2-layer parameters from ionograms based on the empirical orthogonal function (EOF) analysis of ionospheric electron density, Earth, Planets and Space, 59 (1), 51-58.

MCNAMARA, L.F. (2006): Quality figures and errors bars for autoscaled Digisonde vertical incidence ionograms, Radio Science, 41 (4), RS4011, doi: 10.1029/2005RS003440.

Pezzopane, M. (2004): Interpre: a Windows software for semiautomatic scaling of ionospheric parameters from ionograms, Computers \& Geosciences, 30 (1), 125-130.

Pezzopane, M. and C. ScotTo (2004): Software for the Automatic Scaling of Critical Frequency foF2 and MUF(3000)F2 from Ionograms Applied for the Ionospheric Observatory of Gibilmanna, Ann. Geophys.Italy, 47 (6), 1783-1790.

Pezzopane, M. and C. ScotTo (2005): The INGV Software for the Automatic Scaling of Critical Frequency foF2 and $\operatorname{MUF}(3000) \mathrm{F} 2$ from ionograms: a comparison with the ARTIST system 4.01, J. Atmos. Sol. Terr. Phys., 67 (12), 1063-1073.

Pezzopane, M. and C. Scotto (2007): Automatic scaling of critical frequency foF2 and MUF(3000)F2: A comparison between Autoscala and ARTIST 4.5 on Rome data, Radio Science, 42 (4), RS4003, doi: 10.1029/2006RS003581.

REINISCH, B.W. and X. HuANG (1983): Automatic calculation of electron density profiles from digital ionograms 3. Processing of bottomside ionograms, Radio Science, 18 (3), 477-492.

Reinisch, B.W., X. Huang, I.A. Galkin, V. PAZnukhov and A. Kozlov (2005): Recent advances in real-time analysis of ionospheric drift measurements with digisondes, J. Atmos. Sol. Terr. Phys., 67 (12), 1054-1062.

Scotto C. and M. Pezzopane (2002): A software for automatic scaling of foF2 and $\mathrm{MUF}(3000) \mathrm{F} 2$ from ionograms, Proceedings of URSI 2002, Maastricht, 17-24 August 2002, paper 1018 of the electronic copy.

Scotto C. and M. Pezzopane (2007): A method for automatic scaling of sporadic E layers from ionograms, Radio Science, 42 (2), RS2012, doi: 10.1029/2006RS003461.

WAKAI, N., H. OHYAMA and T. KoIZUMI (1987): Manual of Ionogram Scaling, 3rd Version, Radio Research Laboratory Ministry of Posts and Telecommunications, Japan, pp. 119.

(received February 7, 2008; accepted May 9, 2008) 